\title{
Lattice Dynamical Calculations for Inverse Spinel Compounds
}

\author{
A.K. KushWAHA* \\ Department of Physics, K.N. Government P.G. College, Gyanpur, Bhadohi-221 304, India \\ (Received May 15, 2014; in final form January 21, 2015)
}

\begin{abstract}
Lattice dynamical calculations have been performed for inverse spinel structure compounds $\mathrm{MIn}_{2} \mathrm{~S}_{4}(\mathrm{M}=\mathrm{Mn}$ and Co) using the proposed six parameter bond-bending force constant model. In this model, the short-range force constant is calculated by using the Taylor expansion of the potential energy in the harmonic approximation. This model is applied to study the zone-centre $(\Gamma=0)$ phonon frequencies of inverse spinels $\mathrm{MIn}_{2} \mathrm{~S}_{4}(\mathrm{M}=\mathrm{Mn}$ and $\mathrm{Co})$. The significant outcome of the present work is that the second neighbor interactions (octahedral bonding) are stronger than the first neighbor interactions (tetrahedral bonding). The zone-center phonon frequencies, calculated using these parameters, are found to be in very good agreement with the observed results.
\end{abstract}

DOI: 10.12693 /APhysPolA.127.741

PACS: 63.20.-e

\section{Introduction}

The ternary compounds $\mathrm{A}_{2} \mathrm{C}_{4}$ with spinel structure are one of the most important and comprehensively interesting in all structure types. The ferromagnetic properties of some spinels are of great importance in the solid state industry. The structural, vibrational, electrical, dielectric, magnetic properties, solid state reactions and other properties of spinel structure compounds are of major research interest. The lattice dynamical calculations give the detailed information about the vibrational spectra (Raman and infrared), bonding and numerous other solid state properties of spinel structure compounds.

Lattice dynamical calculations for the spinel structure compounds are performed from last forty years [1-13], but most of the calculations are based on the crude models due to the lack of experimental infrared and Raman data. Recently, $a b$ initio calculations have been performed to calculate infrared and Raman phonon modes at the zone centre by Pascal and Gervais [14] and Wijs et al. [15] for $\mathrm{MgAl}_{2} \mathrm{O}_{4}$ and by Fang et al. [16] for $\mathrm{Cu}^{-}$ bic $\mathrm{Si}_{3} \mathrm{~N}_{4}$. Very recently, the ab initio technique has been used to calculate the lattice dynamical properties of $\mathrm{LiMn}_{2} \mathrm{O}_{4}$ by Fang et al. [17].

All the above lattice dynamical calculations are only for the compounds having normal spinel structure. The lattice dynamical calculations for the inverse spinel structure compounds are scarce due to the complexity present in their crystalline structure. Gupta and Balram [18] has calculated the zone-centre phonon frequencies of inverse spinel compounds using an angular force constant model (SRM). They have assumed that the third neighbour interaction is average of the interatomic interaction for the sulphide spinels $[19,20]$ having normal spinel structure and is fixed for all the inverse spinels. Kushwaha [21] has calculated the zone-centre phonons for the inverse spinel compounds $\mathrm{Fe} \mathrm{In}_{2} \mathrm{~S}_{4}$ and $\mathrm{NiIn}_{2} \mathrm{~S}_{4}$ using rigid ion model.

\footnotetext{
*e-mail: akkphys_bu@yahoo.com
}

In the present paper, we have developed a theoretical model to study the zone-centre phonon frequencies of the inverse spinel structure compounds $\mathrm{MIn}_{2} \mathrm{~S}_{4}$ $(\mathrm{M}=\mathrm{Mn}$ and $\mathrm{Co})$. In the present calculations, three bond-stretching, three bond-bending force constants are evaluated by fitting available experimental Raman infrared modes.

\section{Crystal structure and potential model}

The inverse spinel structure compounds $\mathrm{MIn}_{2} \mathrm{~S}_{4}$ $(\mathrm{M}=\mathrm{Mn}$ and $\mathrm{Co})$ belongs to the space group $F d 3 m\left(O_{h}\right)$. In these compounds one half of In ions are on tetrahedral sites and other half of In ions plus M ions occupy octahedral sites randomly. These are represented by $(\operatorname{In})_{\mathrm{T}}(\mathrm{MIn})_{\mathrm{O}} \mathrm{S}_{4}$. The primitive rhombohedral unit cell contains two formula units, i.e., 14 atoms per unit cell.

The 42 dimensional representations of these compounds can be resolved into 17 fundamental lattice vibration modes at the zone center [22-24]. These representations are

$$
\begin{aligned}
\Gamma & =A_{1 \mathrm{~g}}+E_{\mathrm{g}}+2 E_{\mathrm{u}}+2 A_{2 \mathrm{u}}+F_{1 \mathrm{~g}}+3 F_{2 \mathrm{~g}} \\
& +5 F_{1 \mathrm{u}}+2 F_{2 \mathrm{u}} .
\end{aligned}
$$

There are five Raman active $\left(A_{1 \mathrm{~g}}, E_{\mathrm{g}}, 3 F_{2 \mathrm{~g}}\right)$ and four infrared active $\left(4 F_{1 \mathrm{u}}\right)$ modes and rest are Raman inactive $\left(F_{1 \mathrm{~g}}\right)$ and infrared inactive $\left(2 A_{2 \mathrm{u}}, 2 E_{\mathrm{u}}\right.$ and $\left.2 F_{2 \mathrm{u}}\right)$ modes.

The secular determinant is given by

$$
\left|D\left(\boldsymbol{q}, k k^{\prime}\right)-\omega^{2} \delta_{\alpha \beta} \delta_{k k \prime}\right|=0,
$$

where $k k \prime=1,2, \ldots s$ label the ions per unit cell. $\boldsymbol{q}$ denotes the wave-vector whose allowed values range over the first Brillouin zone, $\alpha$ and $\beta=x, y, z$ designate the coordinate axes and $\delta$ is the usual Kronecker delta symbol. The elements of the dynamical matrix are defined as:

$$
\begin{aligned}
& D_{\alpha \beta}\left(\boldsymbol{q}, k k^{\prime}\right)= \\
& \sum_{l^{\prime}} \frac{1}{\sqrt{m_{k} m_{k^{\prime}}}} \varphi_{\alpha \beta}(l k, l / k \prime) \exp \left(\mathrm{i} \boldsymbol{q} \cdot \boldsymbol{r}^{0}(l k, l / k \prime)\right),
\end{aligned}
$$

where $\boldsymbol{r}^{0}(l k, l / k \prime)=\boldsymbol{r}^{0}(l / k \prime)-\boldsymbol{r}^{0}(l k)$ and $m_{k}$ is the mass of the ion $k$-th in the $l$-th cell. As usual $\varphi_{\alpha \beta}(l k, l / k \prime)$ denotes the coupling parameters between ions $l k$ and $l ı k$. 
For the short-range non-Coulombic interaction, the potential energy of the spinel structure $\varphi^{N}$ using Taylor's series can be expressed as

$$
\begin{aligned}
\varphi^{N} & =\sum_{l m n}\left[\frac { 1 } { r } ( \frac { \mathrm { d } \varphi ^ { N } } { \mathrm { d } r } ) _ { | \boldsymbol { r } | = | \boldsymbol { r } _ { k } | } \left\{r_{l m n}^{0}\left(S_{l m n}-S_{0}\right)\right.\right. \\
& \left.+\frac{1}{2}\left|S_{l m n}-S_{0}\right|^{2}\right\}+\frac{1}{2}\left[\frac{1}{r} \frac{\mathrm{d}}{\mathrm{d} r}\left(\frac{1}{r} \frac{\mathrm{d} \varphi^{N}}{\mathrm{~d} r}\right)\right]_{|\boldsymbol{r}|=\left|\boldsymbol{r}_{k}\right|} \\
& \left.\times\left[r_{l m n}^{0}\left(S_{l m n}-S_{0}\right)\right]^{2}\right],
\end{aligned}
$$

where $S_{0}$ and $S_{l m n}$ are the displacements of the central ion and its first neighbor ions from their normal positions, $r_{l m n}$ represents the position coordinates of neighboring ions in equilibrium. $l, m, n$ represent the direction cosines of the line joining the central ion and a nearest neighbor. $\left|\boldsymbol{r}_{k}\right|$ is the nearest neighbor distance.

In our work we have considered the non-Coulombic interaction between central ion and its three nearest neighbors. Let $A_{k}$ be the bond-stretching force constant defined by the second derivative of the potential energy $\varphi^{N}$ :

$$
\frac{e^{2}}{V} A_{k}=\left.\frac{\mathrm{d}^{2} \varphi^{N}}{\mathrm{~d} r^{2}}\right|_{|\boldsymbol{r}|=\left|\boldsymbol{r}_{k}\right|} .
$$

The bond-bending force constant $B_{k}$ is expressed as the first derivative of the potential energy $\varphi^{N}$ :

$$
\frac{e^{2}}{V} B_{k}=\left.\frac{1}{r} \frac{\mathrm{d} \varphi^{N}}{\mathrm{~d} r}\right|_{|\boldsymbol{r}|=\left|\boldsymbol{r}_{k}\right|} .
$$

Here $k=1,2,3$ for first, second and third neighbors.

\section{Results and discussion}

In the present paper, let $A_{1}$ and $B_{1}$ represent the bondstretching and bond-bending force constants between $\mathrm{M}$ and $\mathrm{S}$ type of ions respectively, $A_{2}$ and $B_{2}$ represent similar force constants between In and $\mathrm{S}$ types of ions and $A_{3}$ and $B_{3}$ correspond to bond-stretching and bond-bending force constants between $\mathrm{S}-\mathrm{S}$ types of ions. The dynamical matrix of the order of $42 \times 42$ is obtained and is solved at the zone-centre and gets the analytical expressions for all the different modes.

\section{TABLE I}

The values of force constant (kdynes/cm) for $\mathrm{AIn}_{2} \mathrm{~S}_{4}$, (Mn and Co).

\begin{tabular}{c|c|c}
\hline \hline $\begin{array}{c}\text { Force } \\
\text { parameters }\end{array}$ & $\operatorname{MnIn}_{2} \mathrm{~S}_{4}$ & $\operatorname{CoIn}_{2} \mathrm{~S}_{4}$ \\
\hline$A_{1}$ & 48.34 & 49.74 \\
$B_{1}$ & 1.48 & 1.56 \\
$A_{2}$ & 86.3 & 87.63 \\
$B_{2}$ & 1.71 & 1.83 \\
$A_{3}$ & 12.80 & 13.20 \\
$B_{3}$ & 0.18 & 0.19
\end{tabular}

By using the analytical expressions for the mode $A_{1 \mathrm{~g}}, E_{\mathrm{g}}$ and $F_{1 \mathrm{u}}$ and the corresponding experimental phonon frequencies [25] at the zone-centre, three bondstretching $\left(A_{1}, A_{2}\right.$ and $\left.A_{3}\right)$ and three bond-bending
$\left(B_{1}, B_{2}\right.$ and $\left.B_{3}\right)$ force constants are evaluated and are listed in Table I. Using above force constants the phonon frequencies at the zone-centre for the compounds $\mathrm{MIn}_{2} \mathrm{~S}_{4}$ $(\mathrm{M}=\mathrm{Mn}$ and $\mathrm{Co})$ are calculated.These are listed in Ta-

TABLE II

Measured [25, 26] and calculated zone center phonon frequencies $\left(\mathrm{cm}^{-1}\right)$ of $\mathrm{MnIn}_{2} \mathrm{~S}_{4}$ along with the results of Gupta et al. [18].

\begin{tabular}{c|c|c|c|c}
\hline \hline \multirow{2}{*}{ Species } & \multicolumn{3}{|c}{ Frequencies $\left[\mathrm{cm}^{-1}\right]$} \\
\cline { 2 - 4 } & \multicolumn{2}{|c|}{ Measured } & \multirow{2}{*}{ Calculated } & Gupta et al. \\
\cline { 2 - 4 } & Guc et al. & Lutz et al. & & \\
\hline$A_{1 \mathrm{~g}}$ & 337 & 367 & 340 & 365.9 \\
$E_{\mathrm{g}}$ & 245 & 248 & 246 & 247.3 \\
$F_{2 \mathrm{~g}}(1)$ & 320 & 318 & 321 & 335.2 \\
$F_{2 \mathrm{~g}}(2)$ & 261 & 263 & 262 & 294.8 \\
$F_{2 \mathrm{~g}}(3)$ & 102 & & 100 & 86.7 \\
$F_{1 \mathrm{u}}(1)$ & 303 & 307 & 308 & 314.7 \\
$F_{1 \mathrm{u}}(2)$ & 221 & 216 & 223 & 286.4 \\
$F_{1 \mathrm{u}}(3)$ & 180 & & 179 & 170.2 \\
$F_{1 \mathrm{u}}(4)$ & 87 & & 85 & 85.59 \\
$A_{2 \mathrm{u}}(1)$ & & & 382 & 398.5 \\
$A_{2 \mathrm{u}}(2)$ & & & 203 & 200.6 \\
$E_{\mathrm{u}}(1)$ & & & 368 & 366.7 \\
$E_{\mathrm{u}}(2)$ & & & 133 & 136.4 \\
$F_{1 \mathrm{~g}}$ & & & 246 & \\
$F_{2 \mathrm{u}}(1)$ & & & 349 & 346.7 \\
$F_{2 \mathrm{u}}(2)$ & & & 92 & 93.90 \\
& & &
\end{tabular}

TABLE III

Measured [26] and calculated zone center phonon frequencies $\left(\mathrm{cm}^{-1}\right)$ of $\mathrm{CoIn}_{2} \mathrm{~S}_{4}$ along with the results of Gupta et al. [18].

\begin{tabular}{c|c|c|c}
\hline \hline \multirow{2}{*}{ Species } & \multicolumn{3}{|c}{ Frequencies $\left[\mathrm{cm}^{-1}\right]$} \\
\cline { 2 - 4 } & Measured & Calculated & Gupta et al. \\
\hline$A_{1 \mathrm{~g}}$ & 370 & 368 & 368.9 \\
$E_{\mathrm{g}}$ & 252 & 253 & 251.3 \\
$F_{2 \mathrm{~g}}(1)$ & 315 & 365 & 338.4 \\
$F_{2 \mathrm{~g}}(2)$ & & 278 & 298.3 \\
$F_{2 \mathrm{~g}}(3)$ & & 86 & 87.69 \\
$F_{1 \mathrm{u}}(1)$ & 319 & 322 & 322.2 \\
$F_{1 \mathrm{u}}(2)$ & 235 & 242 & 306.3 \\
$F_{1 \mathrm{u}}(3)$ & 187 & 176 & 168.9 \\
$F_{1 \mathrm{u}}(4)$ & 92 & 89 & 85.7 \\
$A_{2 \mathrm{u}}(1)$ & & 350 & 400.3 \\
$A_{2 \mathrm{u}}(2)$ & & 94 & 204.1 \\
$E_{\mathrm{u}}(1)$ & & 372 & 368.3 \\
$E_{\mathrm{u}}(2)$ & & 152 & 136.7 \\
$F_{1 \mathrm{~g}}$ & & 251 & \\
$F_{2 \mathrm{u}}(1)$ & & 394 & 364.5 \\
$F_{2 \mathrm{u}}(2)$ & & 197 & 89.2
\end{tabular}

ble II and Table III, respectively, along with the available experimental results [25, 26]. This shows a fairly good agreement between theoretical and experimental results. 
From these tabulated force parameters we can conclude that the values of $A_{2}$ (second neighbour interaction) are greater in comparison to $A_{1}$ (first neighbour interaction), i.e., the octahedral bonding is stronger than the tetrahedral bonding. The reason is that the octahedral bonding is more ionic in comparison to the tetrahedral bonding. It is also found that the random occupancy of the octahedral site of inverse spinel compounds does not affect zone-centre phonon frequencies. In the previous lattice dynamical calculations for inverse spinel structure compounds done by Gupta et al. [18], he found 24 infrared phonon modes which differs the number of infrared phonon modes (14 infrared modes) for the normal spinel structure compounds from group theoretical analysis but in present paper we have found 14 infrared phonon modes as found in the normal spinel structure compounds.

\section{Summary}

In the present paper, six parameter bond-bending force constant model is used to calculate the bond strength up to the third nearest neighbours for the inverse spinel structure compounds $\mathrm{MIn}_{2} \mathrm{~S}_{4}(\mathrm{M}=\mathrm{Mn}$ and $\mathrm{Co}$ ). We found that the octahedral bonding (more ionic) is stronger than the tetrahedral bonding (less ionic). We also calculated the zone centre phonon frequencies for $\mathrm{MnIn}_{2} \mathrm{~S}_{4}$ and $\mathrm{CoIn}_{2} \mathrm{~S}_{4}$. We found an overall good agreement with the experimental and theoretical results available in the literature.

\section{References}

[1] G. Harbeke, H. Pinch, Phys. Rev. Lett. 17, 1090 (1966).

[2] M.E. Striefler, G.R. Barsch, J. Phys. Chem. Solids 33, 2229 (1972).

[3] P. Bruesch, F. D'Ambrogio, Phys. Status Solidi B 50, 513 (1972).

[4] H.D. Lutz, H. Haeuseler, Ber. Bunsenges Phys. Chem. 79, 604 (1975).

[5] H. Shimizu, Y. Ohbayashi, K. Yamamoto, K. Abe, J. Phys. Soc. Jpn. 38, 750 (1975).
[6] S.I. Boldish, W.B. White, Rare Earths Mod. Sci. Technol. 13, 607 (1978).

[7] H.A. Lauwers, M.A. Herman, J. Phys. Chem. Solids 41, 223 (1980).

[8] M.A. Aldzhanov, A.M. Aliev, R.K. Veliev, K.K. Mamedov, M.A. Mekhtiev, V.Ya. Shteinshraiber, Phys. Status Solidi B 115, K75 (1983).

[9] M. Wakki, Jpn. J. Phys. Part 1 24, 1471 (1985).

[10] K. Wakamura, H. Iwatani, K. Takarabe, J. Phys. Chem. Solids 48, 857 (1987).

[11] M.E. Striefler, G.R. Barsch, J. Phys. Chem. Solids 48, 57 (1987).

[12] H.C. Gupta, M.M. Sinha, K.B. Chand, Balram, Phys. Chem. Solids 53, 775 (1992).

[13] J. Zwinscher, H.D. Lutz, J. Solid State Chem. 118, 43 (1995).

[14] T. Pascal, F. Gervais, J. Phys. C 14, 3543 (2002).

[15] G.A. de Wijs, C.M. Fang, G. Kresse, G. de With, Phys. Rev. B 65, 094305 (2002).

[16] C.M. Fang, G.A. de Wijs, H.T. Hintzen, G. de With, J. Appl. Phys. 93, 5175 (2003).

[17] C.M. Fang, G.A. de Wijs, C.K. Loong, G. de With, J. Mater. Chem. 17, 4908 (2007).

[18] H.C. Gupta, Balram, J. Phys. Soc. Jpn. 64, 142 (1995).

[19] H.C. Gupta, M.M. Sinha, K.B. Chand, Balram, Phys. Status Solidi B 169, K95 (1992).

[20] H.C. Gupta, M.M. Sinha, K.B. Chand, Balram, J. Phys. Chem. Solids 53, 775 (1992).

[21] A.K. Kushwaha, Indian J. Pure Appl. Phys. 46, 788 (2008).

[22] W.B. White, B.A. De Angelis, Spectrochim. Acta 23A, 985 (1967).

[23] H.D. Lutz, Z. Naturforsch. 24a, 1417 (1969).

[24] A. Chopelas, A.M. Hofmeister, Phys. Chem. Miner. 1, 1861 (1991).

[25] M. Guc, V.V. Ursaki, I.V. Bodnar, D.V. Lozhkin, E. Arushanov, V. Izquierdo-Roca, A. Pérez Rodríguez, Mater. Chem. Phys. 136, 883 (2012).

[26] H.D. Lutz, G. Waschenbach, G. Kliche, H. Heauseler, J. Solid State Chem. 48, 196 (1983). 